\title{
Chorioallantoic Membrane (CAM) Assay of Different Extracts of Rhizome and Inflorescence of Heliconia rostrata
}

\author{
Moonmun Dhara*, Lopamudra Adhikari, Raja Majumder
}

School of Pharmaceutical Sciences, Siksha 'O’ Anusandhan University, Kalinga Nagar, Ghatikia, Bhubaneswar-751003, Odisha, INDIA.

\begin{abstract}
Background: Rhizomes of Heliconia rostrata (Heliconiaceae) has antiophidic, in vitro antioxidant and antibacterial activity. Aim: Extracts of rhizome and inflorescence of $H$. rostrata were studied to test their angiogenesis and anti-angiogenesis effect using ex-ovo chorioallantoic membrane (CAM) assay. Methods: Dried defatted rhizomes of $H$. rostrata were macerated using ethanol, methanol and water to obtain rhizome ethanol extract (REE), rhizome methanol extract (RME) and rhizome aqueous extract (RAE), respectively. Similarly, defatted dried inflorescence of $H$. rostrata was macerated with water to obtain inflorescence aqueous extract (FAE). Dried extracts were used in 0.50 , $1.00,1.50,2.00$ and $2.50 \mathrm{mg}$ dose applied in $3 \mathrm{~mm}$ diameter filter paper for the CAM study. Pictorial as well as a semi-quantitative method was used to evaluate the antiangiogenic effect of the extracts in different doses. Changes before at $\mathrm{Oh}$ and after treatment at $4 \mathrm{~h}$ were compared. Results: The CAM treated with RAE and FAE showed anti-angiogenic effect at $2.50 \mathrm{mg}$ dose per paper. While, CAM treated with REE and RME showed anti-angiogenic effect at $1.50 \mathrm{mg}$ dose per paper. Semi-quantitative evaluation done by five different evaluators (blindly) suggested RME to be the strongest among the extracts in terms of the anti-angiogenic effect. No angiogenic effect is seen. Conclusion: But the higher doses at which the anti-angiogenic effect is seen suggests that Heliconia rostrata may be considered neither angiogenic nor anti-angiogenic.
\end{abstract}

Key words: Rhizome, Inflorescence, Chorioallantoic, Angiogenesis, Anti-angiogenesis.

\section{INTRODUCTION}

According to the Botanical Drug development Guidance, the knowledge of the carcinogenicity of a botanical drug product is one of the key steps in its development as a drug for clinical use. ${ }^{1}$

Many in vivo studies are present that allow the preliminary investigation of carcinogenic potential of a new molecule or drug product without being directly entering into the studies on rodents. One of them is Chick embryo chorioallantoic membrane (CAM) assay. CAM assay has been widely used as an in vivo model to study angiogenic effect, tumor cell invasion and metastasis of many new molecules and herbal preparations. ${ }^{2}$

CAM assay is an in vivo pre-clinical model used for testing the vascular and anti-vascular effects of health care agents. This technique of studying therapeutic agents is not only easy but also is economical in terms of cost and time. It has high reproducibility and gives tissue responses parallel to mammalian models. ${ }^{3}$ Sequencing of chicken genome revealed that it possesses approximately same number of genes as human beings, also with high level of sequence conservation. ${ }^{4} \mathrm{CAM}$ assay is considered to be suitable alternative model for the analysis of acute toxicity of a drug before going for a full scale investigation on the rodents in development of an anticancer agent. ${ }^{1}$ CAM assay not only allows toxicological analysis, but also allows the study of tissue graft, tumour growth,
Submission Date: 02-02-2018; Revision Date: 17-05-2018; Accepted Date: 14-08-2018

DOI: 10.5530/ijper.52.4s.104 Correspondence: Miss. Moonmun Dhara, School of Pharmaceutical Sciences, Siksha 'O' Anusandhan University, Kalinga Nagar, Ghatikia, Bhubaneswar-751003, Odisha, INDIA. Phone: +919040593741 E-mail:moonmundhara@ gmail.com

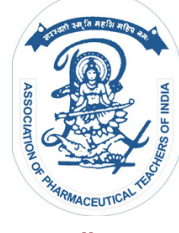

www.ijper.org 
metastasis, wound healing, drug delivery, angiogenesis and anti-angiogenesis of a substance. ${ }^{5}$

Heliconia rostrata (family: Heliconiaceae) is an ornamental herbaceous plant with attractive inflorescence (Figure 1). Humming birds feed on the nectar in the inflorescence which eventually causes pollination. ${ }^{6}$ The rhizomes (Figure 1) of $H$. rostrata have been experimentally proven to have antiophidic property, ${ }^{7}$ in vitro antioxidant and antibacterial activity. ${ }^{8}$ The traditional people of Malaysia (Ipoh, Perak) is said to use the rhizomes of $H$. rostrata to treat intestinal pain, jaundice and high blood pressure. ${ }^{9}$ One of the studies on this plant leaves dealt with the synthesis of gold and silver nanoparticles from the aqueous extract of its leaves and the study of the synthesized nantoparticles for their antiproliferative and apoptoic activity on the EAT cells which resulted in significant reduction in the tumor burden in EAT bearing mice. ${ }^{10}$

The plant $H$. rostrata is considerably new plant in terms of its establishment in the medicinal category. Leaves of this plant successfully showed anticarcinogenic therapeutic potential. ${ }^{10}$

The present work aims at studying the various extracts of rhizome and inflorescence for their angiogenesis and anti-angiogenesis effect using CAM assay so as to prove the therapeutic potential of this plant as a whole.

\section{MATERIALS AND METHODS}

\section{Extraction}

Defatted dried rhizomes of Heliconia rostrata was macerated separately with methanol, ethanol and water for 72 $\mathrm{h}$ to obtain rhizome methanolic extract (RME), rhizome ethanolic extract (REE) and rhizome aqueous extract (RAE). Inflorescence aqueous extract (FAE) was pre-

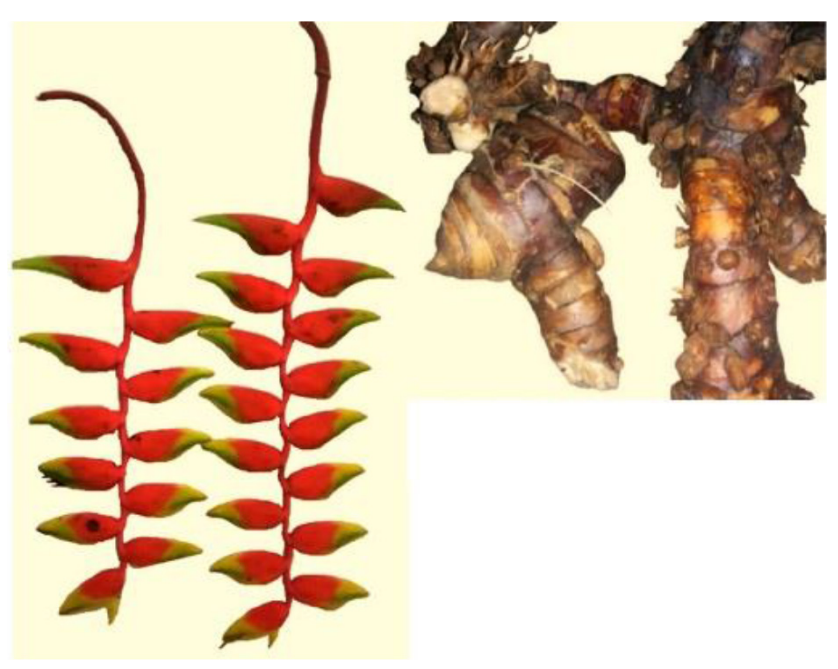

Figure 1: Inflorescence and rhizome of $\boldsymbol{H}$. rostrata. pared by macerating defatted dried inflorescence with water for $72 \mathrm{~h}$. The extracts were filtered and dried under hot water bath to obtain powdered extracts.

\section{Ex-ovo chorioallantoic membrane (CAM) assay}

5 day old fertile chicken eggs incubated at $37^{\circ} \mathrm{C}$ in humidified incubator were obtained from Central Poultry Development Organization (E.R.), Bhubaneswar.

\section{Candling experiment}

It was done to check the viability of the eggs prior to the treatment. Position of the developing embryo was checked using a flashlight. The dead and underdeveloped embryos were discarded. To prevent eggs from getting infected while opening, eggs were first cleaned using $70 \%$ ethanol to remove the dirt and debris. ${ }^{11}$

\section{Preparation of doses}

All the extracts were dissolved in their respective solvents and a concentration of $20 \mathrm{mg} / \mathrm{ml}$ was prepared. REE was dissolved in ethanol; RME was dissolved in methanol and RAE and FAE were dissolved in water. Filter papers of $3 \mathrm{~mm}$ diameter ${ }^{12}$ were punched out and sterilized properly. Different volumes of extract solution were applied drop wise on the sterilized filter papers according to the required dose to be tested. 25 , $50,75,100$ and $125 \mu \mathrm{l}$ were applied on individual paper to attain doses of $0.50,1.00,1.50,2.00$ and $2.50 \mathrm{mg}$, respectively. Testing was done in the ascending order of the doses each time a dose fail to show any change. $125 \mu \mathrm{l}$ of methanol, ethanol and water was applied to filter papers, dried properly and used as negative control.

\section{Approach}

The whole process was performed under the laminar flow. ${ }^{12}$ Eggs were cautiously broken using forceps from the narrow side of the eggs. Then, $2-3 \mathrm{ml}$ albumin was slowly taken out using a syringe with a 21-gauge needle. ${ }^{12}$ The embryo and its extra embryonic membranes were transferred to a petri dish $^{5}$. Dried filtered papers carrying different concentration of extracts were placed carefully on the chorioallantoic membrane for $4 \mathrm{~h}$. Experimentation on each concentration was performed in triplicate. Images of the embryo were captured at $0 \mathrm{~h}$ and at $4 \mathrm{~h}$ of incubation after carefully removing the extract carrying paper. ${ }^{13}$ The images were carefully observed for damage/destruction.

A modified semi quantitative score system (Table 1) was used for the quantification of the damage/destruction by comparing $0 \mathrm{~h}$ (before treatment) and $4 \mathrm{~h}$ (after treatment) images of each dose. ${ }^{12}$ The degree of damage was blindly recorded by five independent observers and an average was calculated. 
Table 1: Semi quantitative scoring system of quantitative estimation of damage/destruction in CAM assay after treatment. ${ }^{9}$

\begin{tabular}{|l|l|}
\hline Scale & Effects observed on CAM after treatment \\
\hline 0 & No change \\
\hline 0.5 & Minor change in the blood vessels \\
\hline 1 & $\begin{array}{l}\text { Small capillary free area below the area of treatment; a few micro } \\
\text { vessels congregate or the growth of blood vessels marginally reduced. }\end{array}$ \\
\hline 2 & $\begin{array}{l}\text { Capillary free area below the area of treatment; micro vessels no } \\
\text { longer visible and large vessel merging. }\end{array}$ \\
\hline
\end{tabular}

\section{RESULTS AND DISCUSSION}

Angiogenesis is an important step in the tissue expansion which when get enhanced leads to the formation of tumors and haematological malignancies. ${ }^{14}$ Anti-angiogenesis therapy is one of the standard care therapies for various types of tumors which act by blocking the formation of new blood vessels in tumor cells. Synthetic drugs like Bevacizumab, Ramucirumab, Axitinib, Sorafenib acts by this mechanism and helps in the treatment of metastatic colorectal cancer, gastroesophageal junction adenocarcinoma, renal cell carcinoma, hepatocellular carcinoma, respectively. ${ }^{15}$

CAM assay is widely used for proangiogenic and antiangiogenic evaluation of different materials. ${ }^{16}$

Allantois of the chick embryo which appears in 3-5 days of incubation pushes out the body of embryo to extra embryonic coelam. From day 4-10, the allantoic vesicle enlarges rapidly and mesodermal layer of the allantois fuses with mesodermal layer of chorion to form chorioallantoic membrane (CAM). ${ }^{4}$

The CAM assay fits in comfortably in the "three R's" strategy of "Principles of Humane Experimental Technique" which was established to reduce the use of animals for the investigation processes. The first " $R$ " refers to the replacement of the animal models with other possible in vitro models. But the use of in vitro models is often not ideal because of the discrepancy between the systemic environments of the systems like vascular system. But, CAM is the outermost extraembryonic membrane that is highly vascularized for gaseous exchange and calcium transportation between the embryo and environment. Thus, this problem of discrepancy is overcome with CAM assay. Though CAM assay is an in vivo model, prior approval from any ethical committee is not necessary for experimentation process. This is because of the fact that CAM assay is performed on the chick embryo which are 4 to 5 days old and the chick embryos younger than day 15 are assumed to experience no pain. This not only simplifies the planning process but also makes this assay less time

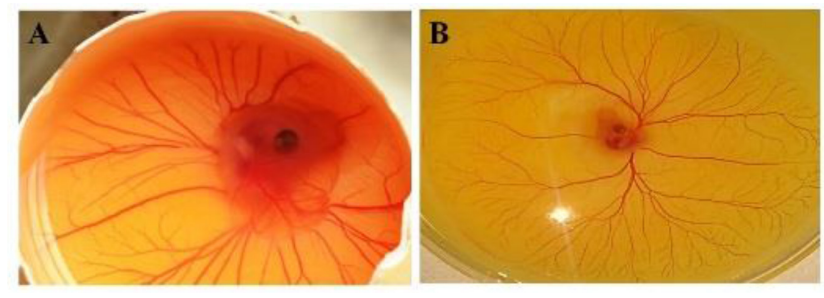

Figure 2: in ovo (A) and ex ovo (B) technique of Chorioallantoic membrane assay (CAM).

consuming. This covers the second " $\mathrm{R}$ " which refers to refinement. The third " $\mathrm{R}$ " is for the reduction of use of animal. Since the prior information of safe dose range is gathered from the CAM assay, wastage of animal life is minimized and the set dose can easily be confirmed with the use of less number of animals. ${ }^{3}$

CAM assay can be conducted either by in ovo technique or ex ovo technique as shown in Figure 2. The in ovo technique (Figure 2A) of CAM assay refers to the assay done through an open window in the egg shell. This technique may preserve the physiological environment and has an advantage when the study period is long. However, this method has major issues with the limits of observation, handling and application of testing reagents. Due to these difficulties, the experimentation here was done with the ex vivo method. In ex vivo technique (Figure 2B), the embryo with the extraembryonic membranes is transferred to a petri dish. This not only provides a two dimensional layer which is easy to visualize for imaging, but also provides an access to the embryo. However, this method can only be used if the study period is kept short. ${ }^{5}$ Since, the study period for this experiment was just $4 \mathrm{~h}$, use of ex vivo method was considered to be the best one in this case.

The different qualitative deviations in the capillaries for each test substances were observed and used to quantify the anti-angiogenic effects of $H$. rostrata rhizome and inflorescence extracts.

It was observed that the area of the CAM below the paper containing methanol, ethanol and water (blank control) did not show any change in the vascular density (Figure 3). Neither growth nor any inhibition was seen with the blank controls. Usual branching pattern of blood vessels in the $4 \mathrm{~h}$ pictures in comparison to the $0 \mathrm{~h}$ pictures indicate that the paper weight and the solvents did not affect the blood vessels.

CAMs treated with different doses of REE, RME, RAE and FAE are depicted in Figure 4 and 5. Comparison of $0 \mathrm{~h}$ pictures in each case is done with $4 \mathrm{~h}$ pictures to find the changes. Inhibition of growth of blood vessels is seen with REE and RME at dose $1.50 \mathrm{mg}$ 


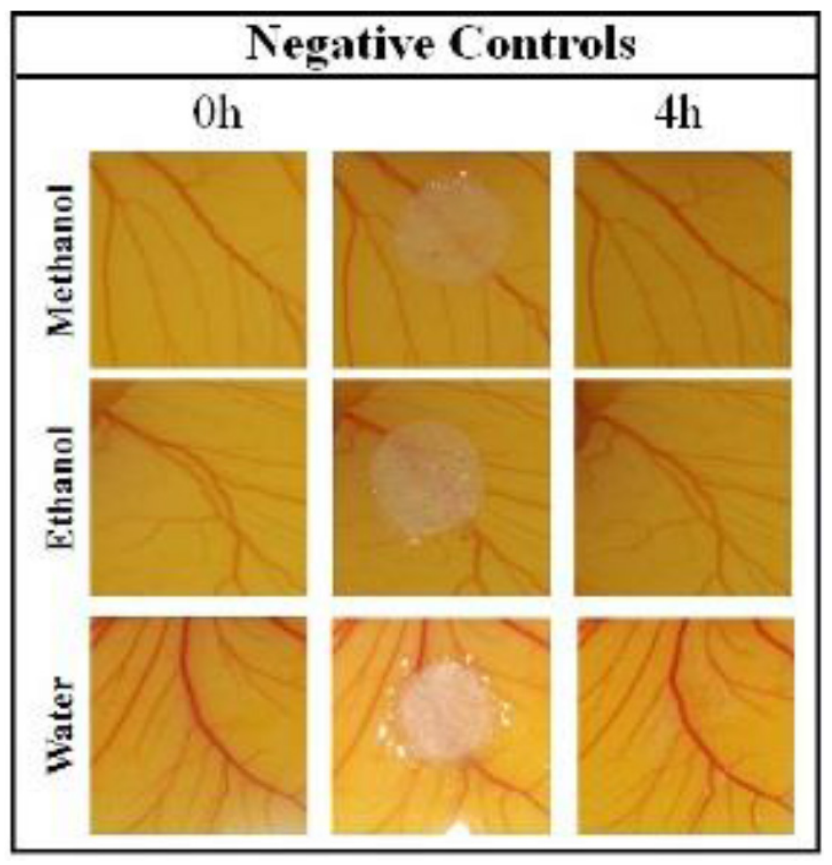

Figure 3: CAM of methanol, ethanol and water, the solvents used to dissolve the extracts.

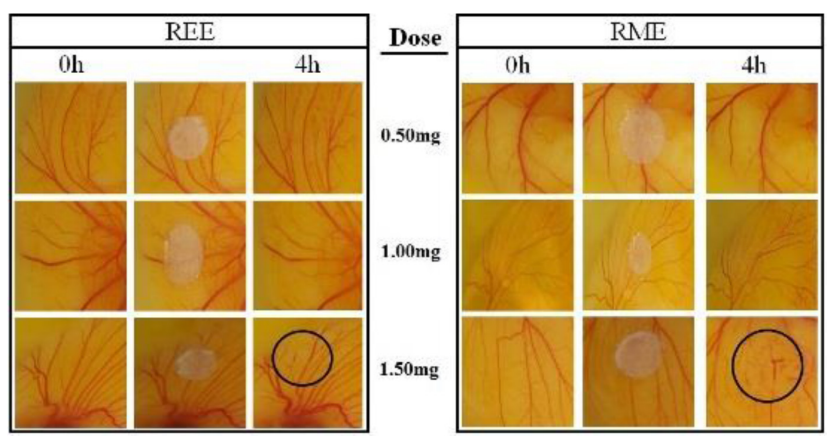

Figure 4: CAM assay of REE and RME at different doses. Damaged/destroyed blood vessels are marked in circle.

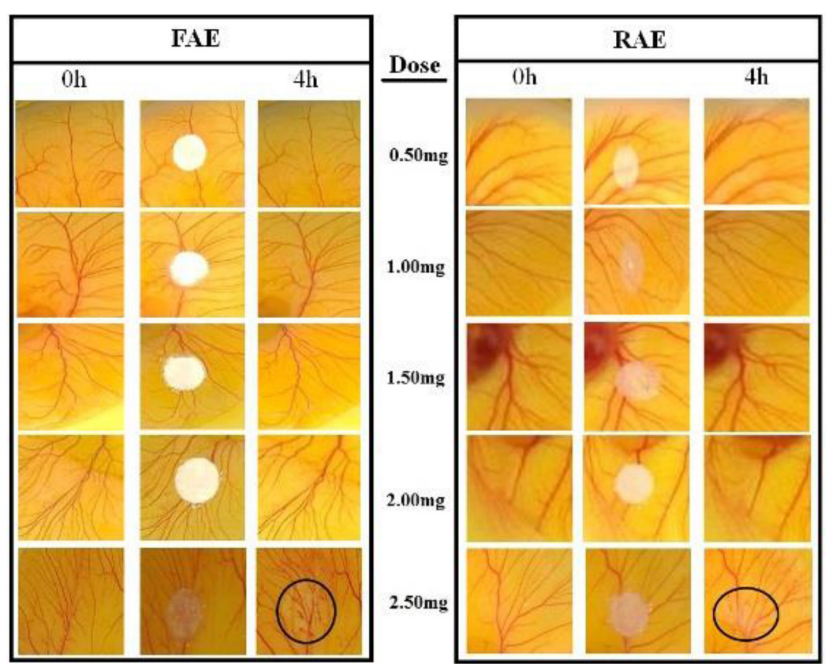

Figure 5: CAM assay of FAE and RAE at different doses. Damaged/destroyed blood vessels are marked in circle.
(Figure 4). No change is observed with the lower doses, $1.00 \mathrm{mg}$ and $0.50 \mathrm{mg}$ of REE and RME. In case of RAE and FAE, damaged or destroyed blood vessel is seen at dose $2.50 \mathrm{mg}$ (Figure 5). Lower doses, $2.00 \mathrm{mg}, 1.50 \mathrm{mg}$, $1.00 \mathrm{mg}$ and $0.50 \mathrm{mg}$ show no change. The branching pattern of blood vessels and the pre-existing vessels was reduced, with dose $1.50 \mathrm{mg}$ for REE and RME (Figure 4) and $2.50 \mathrm{mg}$ for FAE and RAE (Figure 5). Almost capillary free area is observed with the RME, $1.50 \mathrm{mg}$ (Figure 4). The degree of damage was evaluated by average scores calculated using the pictorial observations. This semiquantitative method was based on the observations made by five different observers having no idea of doses and extracts during observations and scores. The scoring was done according to the score pattern in Table 1 ranging from 0 to 2 . Table 3 depicts the average score and the interpretation thus made according to Table 2. RME shows strong anti-angiogenic effect than REE at dose $1.5 \mathrm{mg}$ per disc which shows good anti-angiogenic effect while at $2.5 \mathrm{mg}$ dose both RAE and FAE show weak anti-angiogenic effect.

Angiogenesis refers to the development of new blood vessels from the predating vasculature, ${ }^{12}$ whereas antiangiogenesis refers to the inhibition of growth or death of the blood vessels. Both angiogenesis and anti- angiogenesis have their merits as well as demerits. Angiogenesis plays an important role in growth and metastasis of tumors and thus, cancer. ${ }^{17}$ Here, anti-angiogenic activity is considered to be a promising approach for treatment of cancer. ${ }^{18}$

\section{CONCLUSION}

The Chorioallantoic membrane assay of REE, RME, RAE and FAE at different doses was performed using ex-ovo method. The method was adopted to check for the anti-angiogenic effect of the extracts so as to establish the fact that whether or not said extracts of $H$. rostrata be used for the treatment of cancer. Taking in consideration the observations made from the pictorial study and average scores (Table 2), both RAE and FAE

Table 2: Interpretation of anti-angiogenic effect to be drawn from the average scores of semi-quantitative scoring systems.

\begin{tabular}{|l|l|}
\hline $\begin{array}{c}\text { Average } \\
\text { score }\end{array}$ & \multicolumn{1}{c|}{ Conclusions } \\
\hline$<0.5$ & No anti-angiogenic effect \\
\hline$\geq 0.5$ and $\leq 1$ & Weak anti-angiogenic effect \\
\hline$>1$ and $<1.5$ & Good anti-angiogenic effect \\
\hline$\geq 1.5$ & strong anti-angiogenic effect \\
\hline
\end{tabular}




\begin{tabular}{|}
$\begin{array}{c}\text { Table 3: Anti-angiogenic effects of different extracts } \\
\text { of Heliconia rostrata by CAM assay. }\end{array}$ \\
\hline \multirow{4}{*}{ Extracts } & Dose $(\boldsymbol{\mu g})$ & Average score & $\begin{array}{c}\text { Anti-angiogenic } \\
\text { effect }\end{array}$ \\
\hline \multirow{4}{*}{ REE } & $0.50 \mathrm{mg}$ & $0.00 \pm 0.00$ & No \\
\cline { 2 - 4 } & $1.00 \mathrm{mg}$ & $0.20 \pm 0.24$ & No \\
\cline { 2 - 4 } & $1.50 \mathrm{mg}$ & $1.40 \pm 0.49$ & Good \\
\hline \multirow{4}{*}{ RME } & $0.50 \mathrm{mg}$ & $0.00 \pm 0.00$ & No \\
\cline { 2 - 4 } & $1.00 \mathrm{mg}$ & $0.20 \pm 0.24$ & No \\
\cline { 2 - 4 } & $1.50 \mathrm{mg}$ & $1.60 \pm 0.49$ & Strong \\
\hline \multirow{4}{*}{ FAE } & $0.50 \mathrm{mg}$ & $0.00 \pm 0.00$ & No \\
\cline { 2 - 4 } & $1.00 \mathrm{mg}$ & $0.00 \pm 0.00$ & No \\
\cline { 2 - 4 } & $1.50 \mathrm{mg}$ & $0.00 \pm 0.00$ & No \\
\cline { 2 - 4 } & $2.00 \mathrm{mg}$ & $0.20 \pm 0.24$ & No \\
\cline { 2 - 4 } & $2.50 \mathrm{mg}$ & $0.90 \pm 0.20$ & Weak \\
\hline \multirow{4}{*}{ RAE } & $0.50 \mathrm{mg}$ & $0.00 \pm 0.00$ & No \\
\cline { 2 - 4 } & $1.00 \mathrm{mg}$ & $0.00 \pm 0.00$ & No \\
\cline { 2 - 4 } & $1.50 \mathrm{mg}$ & $0.00 \pm 0.00$ & No \\
\cline { 2 - 4 } & $2.00 \mathrm{mg}$ & $0.10 \pm 0.20$ & No \\
\cline { 2 - 4 } & $2.50 \mathrm{mg}$ & $0.80 \pm 0.24$ & Weak \\
\hline
\end{tabular}

Values are mean \pm Standard deviation $(S D)(n=5)$

are weakly anti-angiogenic and RME is the strongest among all the tested materials. But, the dose at which RME shows the anti-angiogenic effect is very high $(1.50 \mathrm{mg})$ in comparison to Suramin $(50 \mu \mathrm{g})$, an already established drug used as a standard. ${ }^{9}$ Moreover, none of the extracts show any angiogenesis. Thus, all the extracts tested can be considered neutral both in terms of their angiogenesis as well as anti-angiogenic effect. Since, CAM is also used to study the acute toxicity of a substance, ${ }^{1}$ the higher doses of the extracts showing anti-angiogenesis can also fruitfully help in predicting the high $\mathrm{LD}_{50}$ values of the extracts in terms of their toxicity. The toxicity aspect may further be clarified by performing the acute toxicity study on the rodents.

\section{ACKNOWLEDGEMENT}

Authors are thankful to School of Pharmaceutical Sciences, SOA University for providing necessary facilities related to the present research work. Authors also would like to thank Central Poultry Developmental Organization, Bhubaneswar for providing eggs for the study.

\section{CONFLICT OF INTEREST}

The Authors declare no conflict of interest.

\section{ABBREVIATIONS}

h: hour; ml: mililiter; mg: milligram.

\section{REFERENCES}

1. Botanical Drug Development Guidance for Industry. U.S. Department of Health and Human Services. Food and Drug Administration, Center for Drug Evaluation and Research. 2016.

2. Lokman NA, Eldder ASF, Ricciardelli C, Oehler MK. Chick chorioallantoic membrane (CAM) assay as an in vivo model to study the effect of newly identified molecules on Ovarian Cancer Invasion and Metastasis. Int $\mathrm{J}$ of Mol Sci. 2012;13(8):9959-70.

3. Kue CS, Tan KY, Lam ML, Lee HB. Chick embryo chorioallantoic membrane (CAM): an alternative predictive model in acute toxicological studies for anticancer drugs. Exp Anim. 2015;64(2):129-38.

4. Ribatti D. The chick embryo chorioallantoic membrane (CAM) assay. Reprod Toxicol. 2017;70:97-101.

5. Ribatti D. The chick embryo chorioallantoic membrane (CAM). A multifaceted experimental model. Mech Develop. 2016;141:70-7.

6. Hanisworth FR. On the tongue of a hummingbird: Its role in the rate and energetics of feeding. Comp Biochem Phys A. 1973;46(1):65-78.

7. Estrada GS, Jimenez SL, Alarcon PJC, Vargas LJ. Application of ultrasound in the dissolution of potential antiophidian compounds from two ethanolics extracts of two species of Heliconias. Ultrason Sonochem. 2010;17(5):756-9.

8. Moonmun D, Majumder R, Lopamudra A. Quantitative phytochemical estimation and evaluation of antioxidant and antibacterial activity of methanol and ethanol extracts of Heliconia rostrata. Indian J of Pharm Sci. 2017;79(1):79-90.

9. Gunjan M, Karna L, Dayalan K, Sasigaran P. A review and search of Phytomedicine used by traditional people of Malaysia (Ipoh, Perak). International Journal of Phytotherapy Research. 2012;2(3):26-41.

10. Mallesha L, Vinay G, Rekha ND. Synthesis of metal nanoparticles using Heliconia rostrata leaf extract and their antiproliferative and apoptotic property. Current chemistry Letters. 2018;7(2):65-72.

11. Gamallo JPM, Espere G, Carillo DMC, Blanes DN, Abuda FG, Labarda HJ, et al. Evaluation of anti-angiogenic property of Ocimum basilica ethanolic leaf extract by using duck embryo chorioallantoic membrane (CAM) assay and its morphometric analysis. Int J of Herbal Med. 2016;4(4):22-6.

12. Seow L, Beh H, Majid AMSA, Murugaiyah V, Ismail N, Asmawi MZ. Antiangiogenic activity of Gynura segetum leaf extracts and its fractions. J Ethnopharmacol. 2011;134(2):221-7.

13. Nagababu P, Barui AK, Thulasiram B, Devi CS, Satyanarayana S, Patra $\mathrm{CR}$, et al. Antiangiogenic activity of mononuclear Copper (II) Polypyridyl complexes for the treatment of cancers. J Med Chem. 2015;58(13):5226-41.

14. Chen H, Wang CS, Li M, Sanchez E, Li J, Berenson A, et al. A novel angiogenesis model for screening anti-angiogenic compounds: The chorioallantoic membrane/feather bud assay. Int J Oncol. 2010;37(1):71-9.

15. Yang $\mathrm{W}, \mathrm{Xu} \mathrm{J,} \mathrm{Mu} \mathrm{J,} \mathrm{Xie} \mathrm{J.} \mathrm{Revision} \mathrm{of} \mathrm{the} \mathrm{concept} \mathrm{of} \mathrm{anti-angiogenesis}$ and its applications in tumor treatment. Chronic Diseases and Translational Medicine. 2017;3(1):33-40.

16. Jadhav J, Mane A, Kanase A. Antiangiogenic properties of Boerhaavia diffusa extracts in chick Chorioallantoic Membrane (CAM). International Journal of Drug Development and Research. 2011;3(4):307-31.

17. Hazel SJ. A novel early chorioallantoic membrane assay demonstrates quantitative and qualitative changes caused by antiangiogenic substance. $J$ Lab Clin Med. 2003;141(3):217-28.

18. Mathur R, Gupta SK, Singh N, Mathur S, Kochupillai V, Velpandian T. Evaluation of the effect of Withania somnifera root extracts on cell cycle and angiogenesis. J Ethanopharamcol. 2006;105(3):336-41. 


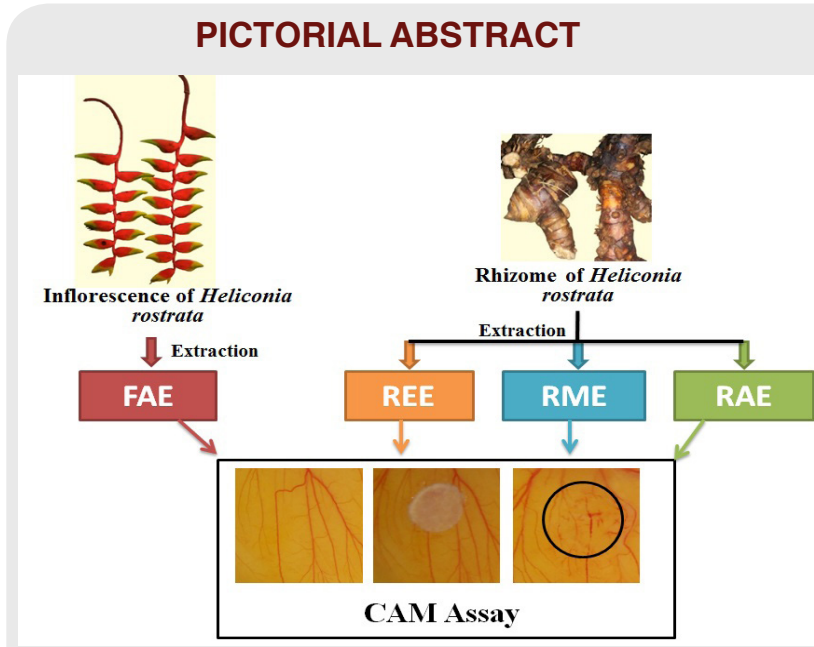

\section{About Authors}

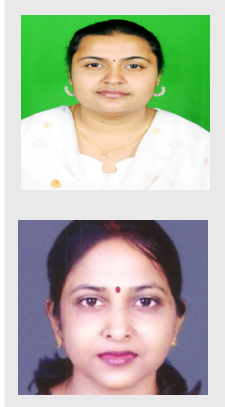

Moonmun Dhara: Research Scholar, School of Pharmaceutical Sciences, Siksha O Anusandhan (Deemed to be University), Bhubaneswar, Odisha, India

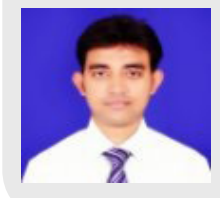

Dr. Lopamudra Adhikari: Associate Professor, Department of Pharmaceutical Analysis and Quality Assurance, School of Pharmaceutical Sciences, Siksha O Anusandhan (Deemed to be University), Bhubaneswar, Odisha, India.

Mr. Raja Majumder: Research Scholar, School of Pharmaceutical Sciences, Siksha O Anusandhan (Deemed to be University), Bhubaneswar, Odisha, India 